\section{Hohes Risiko: Androgene und Brachytherapie optimieren die Bestrahlung}

Ein lokalisiertes Prostatakarzinom ist nur selten eine tödliche Diagnose. Für Patienten mit hohem Progressionsrisiko fehlt aber bisher ein geeigneter Behandlungsstandard. Kombinationen moderner Behandlungsansätze wurden in einer retrospektiven Analyse betrachtet.

A uch wenn viele Krebserkrankungen der Prostata milde verlaufen, gibt es eine Zahl von Patienten mit initial sehr fortgeschrittenen Tumoren. Wie sind sie am besten zu behandeln? Ansätze wie die externe Strahlentherapie (EBRT) mit konventionellen Dosen ( $<72$ Gy), die Brachytherapie sowie die Androgen-Deprivations-Therapie (ADT) erzielten als Monotherapien in der Hochrisiko-Situation jeweils nur unbefriedigende Ergebnisse.

Im Gegensatz dazu wurde durch die Kombination von EBRT und ADT in randomisierten klinischen Studien ein verbessertes Gesamtüberleben erreicht. Ergebnisse einer Subgruppenanalyse legen nahe, dass auch eine dosiseskalierende EBRT die Prostatakarzinom-spezifische Mortalität senken kann. Der Frage, ob auch die Addition einer Brachytherapie oder einer ADT zur Radiotherapie weiteren Benefit erzielt, wurde in einer retrospektiven Studie nachgegangen.

Zwischen 1995 und 2010 wurden 958 Hochrisiko-Patienten mit PSA-Werten über $20 \mathrm{ng} / \mathrm{ml}$, einer Gleasonsumme von 8-10 und klinischen T3-oder T4-Tumoren an zwei US-Kliniken behandelt. Sie erhielten entweder eine dosiseskalierende EBRT ( $\mathrm{n}=510)$ mit mindestens $75 \mathrm{~Gy}$ oder eine „Combined-Modality Radiotherapy“ (CMRT) mit ${ }^{103} \mathrm{Pd}$ - $(\mathrm{n}=369)$ oder ${ }^{125}$ I-Implantaten $(n=79)$ jeweils mit pelviner Bestrahlung (mediane verschriebene Dosis: 45 Gy). Endpunkte waren biochemisches Versagen und Prostatakrebs-spezifische Mortalität.

Das mediane Follow-up betrug 63,2 Monate, 250 Patienten wurden für mehr als acht Jahre beobachtet. Verglichen mit der CMRT-Gruppe zeigten die Patienten der EBRT-Gruppe höhere PSA-Level, fortgeschrittenere Tumoren und eine niedrigere Gleason-Summe; sie erhielten auch häufiger und länger eine ADT. Nach acht Jahren lag die Inzidenz biochemischen Versagens in der EBRTGruppe bei $40 \%$, die Prostatakrebs-spe- zifische Mortalitätsrate bei $13 \%$. In der CMRT-Gruppe betrugen diese Werte 14 bzw. $7 \%$. In der multivariaten Analyse lag die Hazard Ratio für biochemisches Versagen bei 0,35, für die Prostatakrebsspezifische Mortalität bei 0,41 - jeweils zugunsten der CMRT. Wurde die Dauer der ADT erhöht, nahmen biochemisches Versagen und krebsspezifische Mortalität ab - sogar in der CMRT-Subgruppe.

Fazit: In diesem retrospektiven Vergleich senkte eine CMRT mit niedrigdosiertem Brachytherapie-Boost die Raten an biochemischem Versagen und krebsspezifischer Mortalität im Vergleich zur EBRT. Zudem wirkte sich eine ADT bei beiden Modalitäten günstig auf diese Parameter aus - mit dem größten Nutzen bei einer Langzeit-ADT. Sollte sich dies bestätigen, könnte sich der Behandlungsstandard für Hochrisiko-Patienten bald ändern.

Christina Berndt

Shilkrut M et al. The addition of low-dose-rate brachytherapy and androgen-deprivation therapy decreases biochemical failure and prostate cancer death compared with dose-escalated external-beam radiation therapy for high-risk prostate cancer. Cancer. 2012; Aug 14. [Epub ahead of print]

Kommentar von Dr. Zimmermann: Die Autoren haben die Frage, welche Therapie im Hochrisiko-Stadium eines Prostatakarzinomes effektiv ist, in intelligenter Weise beantwortet. Ausgehend vom (leitliniengerechten) Standard einer alleinigen perkutanen Bestrahlung mit mindestens 75-78 Gy GHD wurden sowohl der Einfluss einer zusätzlichen LDR-Brachytherapie (Kombinationsbestrahlung) wie auch einer zusätzlichen Hormontherapie untersucht.

Nach einer medianen Nachbeobachtung von 63 Monaten wiesen die Patienten, die in Kombination mit einer externen Bestrahlung eine zusätzliche LDR-Brachytherapie erhalten hatten, eine erkennbar niedrigere biochemische Progressrate (14 vs. $40 \%$ ) und eine ebenfalls erkennbar geringere PCAMortalitätsrate (7 vs. $13 \%$ ) auf. Die zusätzliche Langzeitgabe einer Hormonblockade verlängerte sowohl die biochemische Progressrate als auch vor allem das Gesamtüberleben weiter signifikant.

Während die zusätzliche LDR-Brachytherapie vor allem an den bekannten strahlenbiologischen Mechanismen der Dosis-Volumen-Effekte ansetzt (viel Dosis hilft viel), ist der hier berichtete Einfluss einer zusätzlich applizierten Hormontherapie sicher differenzierter zu betrachten. Zum einen verlängert sie die Zeit der primären therapieassoziierten PSA-Suppression und trägt, da es erst ab dem Zeitpunkt des Hormontherapieabsetzens zu einem PSA-Anstieg kommen kann, sicher darüber zu einem verlängerten Intervall bis zur Diagnosestellung (PSA-Nadir +2) bei. Dies ist primam vistam nur ein statistischer Effekt, der entgegen den meisten bisher berichteten Beobachtungen in der vorliegenden Studie auch einen Einfluss auf das PCA-assoziierte Überleben hat. Eine Verlängerung der Beobachtungsdauer kann hierüber Aufschluss geben.

Andererseits kann es bei einer ausreichend früh einsetzenden neoadjuvanten Hormontherapie auch zu einer deutlichen Verkleinerung des intraprostatischen Tumorvolumens kommen. Über die schon beschriebenen Mechanismen der Dosis-Volumen-Effekte wäre dann die Erklärung ableitbar, dass verkleinerte Tumoren auf eine gleichbleibende Strahlendosis besser ansprechen als Tumoren, deren Volumen nicht vorher möglichst weit reduziert wurde.

Unabhängig von Einzelheiten sind die insgesamt erreichten geringen Progressund Mortalitätskennzahlen hervorragend und lassen neben der ausgezeichneten Ergebnisse der Metaanalyse von Peter Grimm und Kollegen [Grimm P et al. BJU Int. 2012; 109(Suppl 1):22-9] zu diesem Thema die Hoffnung zu, dass die Kombinationsbehandlung aus externer Bestrahlung und LDR-Brachytherapie eines Tages auch in den deutschen Leitlinien zu einer der Standardbehandlungen wird.

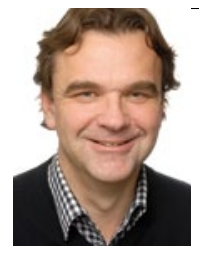

Dr. med. Jörg Zimmermann Arzt für Strahlentherapie Schwerpunkt Brachytherapie info@brachytherapiehamburg.de 